\title{
Aluminum Silicate Nanotube Modification of Cotton-Like Siloxane-poly(L-lactic acid)-vaterite Composites
}

\author{
Daiheon Lee, ${ }^{1}$ Hirotaka Maeda, ${ }^{2}$ Akiko Obata, ${ }^{1}$ Keiichi Inukai, ${ }^{3}$ \\ Katsuya Kato, ${ }^{4}$ and Toshihiro Kasuga ${ }^{1}$ \\ ${ }^{1}$ Department of Frontier Materials, Nagoya Institute of Technology, Gokiso-cho, Showa-ku, Nagoya, Aichi 466-8555, Japan \\ ${ }^{2}$ Center for Fostering Young and Innovative Researchers, Nagoya Institute of Technology, Gokiso-cho, Showa-ku, \\ Nagoya 466-8555, Japan \\ ${ }^{3}$ Ecological Ceramics Group, Materials Research Institute for Sustainable Development, National Institute of Advanced \\ Industrial Science and Technology, Anagahora Shimo-Shidami, Moriyama-ku, Nagoya 463-8560, Japan \\ ${ }^{4}$ Applied Technology with Traditional Ceramics Group, Advanced Manufacturing Research Institute, National Institute of Advanced \\ Industrial Science and Technology, Anagahora Shimo-Shidami, Moriyama-ku, Nagoya 463-8560, Japan
}

Correspondence should be addressed to Hirotaka Maeda; maeda.hirotaka@nitech.ac.jp and Toshihiro Kasuga; kasuga.toshihiro@nitech.ac.jp

Received 17 May 2013; Revised 31 August 2013; Accepted 2 September 2013

Academic Editor: Delia Brauer

Copyright (C) 2013 Daiheon Lee et al. This is an open access article distributed under the Creative Commons Attribution License, which permits unrestricted use, distribution, and reproduction in any medium, provided the original work is properly cited.

In our earlier work, a cotton-like biodegradable composite, consisting of poly(L-lactic acid) with siloxane-containing vaterite, has been prepared by electrospinning. In the present work, the fibers skeleton of the cotton-like composites was modified successfully with imogolite, which is hydrophilic and biocompatible, via a dip process using ethanol diluted solution to improve the cellular initial attachment. Almost no change in the fiber morphology after the surface modification was observed. The surface-modified composite showed the similar calcium and silicate ions releasabilities, for activating the osteoblasts, as an unmodified one. Cell culture tests showed that the initial adhesion of murine osteoblast-like cells on the surface of the fibers was enhanced by surface modification.

\section{Introduction}

Recently, the biodegradable polymers have attracted much attention as scaffolds [1,2]. Poly(L-lactic acid) (PLLA), one of the common biodegradable polymers, has been reported as a scaffold material for bone reconstruction [3]. Several kinds of inorganic ions released from materials are reported to influence osteogenesis and angiogenesis [4]. Xynos et al. suggested that the ionic products, such as calcium and silicate ion released from Bioglass 45S5, have a stimulatory effect on osteoblast proliferation by increasing availability of insulin-like growth factor-II (IGF-II) [5]. The preparation of composites including inorganic materials, with calcium and silicate species releasing ability, in the biodegradable polymers is one of the important technologies for developing bone-forming biomaterials.

Our group has developed a polymer-ceramic composite material, PLLA/siloxane-containing vaterite $(\mathrm{SiV})$ composite
(PLLA/SiV), for the bone-filling material applications. The $\mathrm{PLLA} / \mathrm{SiV}$ has the calcium and silicate ions releasing abilities derived from $\mathrm{SiV}$ [6]. In our earlier works, the PLLA/SiV fiber mats of $\sim 300 \mu \mathrm{m}$ in thickness, fabricated by electrospinning, showed bone formation by implanting the fiber mats on defects of $8 \mathrm{~mm}$ in diameter in the front midline of the calvaria in New Zealand rabbits $[7,8]$. We also have prepared the cotton-like PLLA/SiV consisting of fibrous skeletons with open pore structure successfully by modified-electrospinning $[9,10]$. The cells penetrated into the cotton-like PLLA/SiV and adhered on the fiber surface, resulting in proliferation inside of the cotton-like structure [10].

We believe that the enhancement of cell adhesion on the fiber surface is a critical factor for improving its biocompatibility. It has been widely accepted that the bone-forming cells prefer to attach on hydrophilic surface than hydrophobic one [11-13]. Nanometer-sized hydrous aluminum silicate, which 
is called imogolite, $(\mathrm{HO})_{3} \mathrm{Al}_{2} \mathrm{O}_{3} \mathrm{Si}(\mathrm{OH})$, has tubular structure of $\sim 2.3 \mathrm{~nm}$ external and $\sim 1.0 \mathrm{~nm}$ internal diameters [14], with up to several micrometers lengths. Imogolite nanotubes (INTs) have very hydrophilic surface owing to numerous hydroxyl groups on the surface. Ishikawa et al. reported that human and mouse osteoblast-like cells were widely spread on INTs coated on polystyrene cell culture dish, compared with the culture dish [15-17]. INT is one of the potential candidates to modify the fiber surface in the cotton-like PLLA/SiV for enhancing their cellular attachments.

The calcium and silicate ions releasing ability of PLLA/ $\mathrm{SiV}$, which has an effect on enhancing the cellular activity [18], is very important for accelerating bone formation on the material. Fujikura et al. suggested that the hydroxyapatite coating layer on fibers of the fiber mats, which were prepared by electrospinning, controlled calcium and silicate ions release from the fibers [19]. The influence of INT modification on the ions releasing ability of the cotton-like PLLA/SiV is considered to be examined.

In our previous report, it has been reported that the cell compatibility of PLLA/SiV fiber mats, especially at the early stage, was improved with INTs coating by electrophoretic deposition [20]. In our preliminary experiments, the electrophoretic deposition method has almost no effect on cotton-like PLLA/SiV. INTs would pass over the cotton-like PLLA/SiV because of the very high porosity of cotton-like structure. The aim of this work is to examine the effective way of PLLA/SiV surface modification with INTs. The PLLA/SiV has hydrophobic surface due to the matrix PLLA. INTs are dispersed in aqueous solution as a result of synthesis. It might lead to difficulty of coating on hydrophobic PLLA/SiV surface. It was considered that, by improving hydrophilicity of the fibrous PLLA/SiV surface temporarily, the modification using dip-coating would be able to be applied effectively. Our idea for improving the hydrophilicity is to treat the cotton-like PLLA/SiV with diluted ethanol. In the present work, the dip-coating method, one of the simplest ways, was investigated for INT modification of cotton-like PLLA/SiV to enhance the cellular attachment.

\section{Materials and Methods}

2.1. Preparation of Cotton-Like PLLA/SiV. SiV particles, which were spherical particles of $\sim 1 \mu \mathrm{m}$ diameter, containing $2.6 \mathrm{wt} \%$ silicon, were prepared by a carbonation method using methanol and $\gamma$-aminopropyltriethoxysilane (APTES; Momentive Performance Materials, Japan). The detail of the preparation was described in our previous reports $[7,8$, 18]. PLLA/SiV was prepared by kneading PLLA (LACIA; molecular weight $=\sim 140 \mathrm{kDa}$, Mitsui Chemicals, Japan) with $\mathrm{SiV}$ particles at $200^{\circ} \mathrm{C}$ for $10 \mathrm{~min}$. The weight ratio of PLLA : SiV was $7: 3$.

The PLLA/SiV solution for electrospinning was prepared using a chloroform as a solvent; PLLA weight ratio in the solution was $10 \mathrm{wt} \%$. Following our previous report [9], cotton-like PLLA/SiV was prepared by electrospinning in ethanol (Wako Pure Chemicals, Japan) as a collector at room temperature. We have reported that PLLA fiber mats composed of the fiber skeleton $\sim 10 \mu \mathrm{m}$ in diameter allow cellular ingrowth into the gaps between them [21]. The fibers diameter was controlled by electrospun conditions as follows: impressed voltage: $15 \mathrm{kV}$, collector distance: $300 \mathrm{~mm}$, and solution flow rate: $0.25 \mathrm{~mL} / \mathrm{min}$.

2.2. Preparation of INT-Dispersed Aqueous Solutions. The preparation procedure of INTs was described in our previous report [20]. In brief, $18.47 \mathrm{~g}$ of aluminum chloride $\left(\mathrm{AlCl}_{3}\right.$. $6 \mathrm{H}_{2} \mathrm{O}$; Wako Pure Chemicals, Japan) and $9.20 \mathrm{~g}$ sodium silicate $\left(\mathrm{Na}_{4} \mathrm{SiO}_{4} \cdot \mathrm{nH}_{2} \mathrm{O}\right.$; Wako Pure Chemicals, Japan) were dissolved in $500 \mathrm{~g}$ of distilled water, respectively; Si/Al molar ratio of 0.41 . sodium hydroxide $(\mathrm{NaOH}, 1 \mathrm{~mol} / \mathrm{L}$; Wako Pure Chemicals, Japan) aqueous solution was slowly added to the above described sodium silicate/aluminum chloride solution until $\mathrm{pH}$ of the solution reached 6.8. The resulting samples were separated by centrifugation, and the obtained precipitates were rinsed with distilled water. The centrifugationrinsing process was repeated three times, and then the aluminum silicate precursors were dispersed in $12 \mathrm{~L}$ of distilled water by acidification with hydrochloric acid $(\mathrm{HCl}, 5 \mathrm{~mol} / \mathrm{L}$; Wako Pure Chemicals, Japan). The precursor solution was heated at $95^{\circ} \mathrm{C}$ for 4 days. Finally, the dispersed INTs in an aqueous solution with a concentration of $0.087 \mathrm{wt} \%$ were obtained.

2.3. INT Modification of the Cotton-Like PLLA/SiV by a Dip-Coating Method. The INT-dispersed aqueous solution was diluted with ethanol to prepare the suspension for the dipping process. The weight ratio of INT-dispersed aqueous solution : ethanol was $10: 90$ or $50: 50$. It is well known that the vaterite of $\mathrm{SiV}$ is in the unstable phase in water and easily transforms into calcite [22, 23]. Ethanol was used for dilution because the vaterite is known to be more stable in ethanol than in water [24]. Cotton-like PLLA/SiV was dipped into the suspension and dried in a vacuum chamber at room temperature. The sample, which was modified using 50\% diluted suspension, was denoted as PLLA/SiV-INT(50-1). In the case of $90 \%$ diluted suspension, the sample was prepared by the dipping process by repeating it 5 times and was denoted as PLLA/SiV-INT(10-5), hereafter.

2.4. Evaluation of INT-Modified Cotton-Like PLLA/SiV. The morphology of cotton-like PLLA/SiV fibers was observed by field emission gun scanning electron microscopy (FE-SEM) (JSM-6301F, JEOL, Japan), incorporating energy dispersive X-ray spectroscopy (EDS) (Vantage, Noran, USA), after coating with amorphous osmium by plasma chemical vapor deposition.

The static water contact angles on the pressed samples, by uniaxial pressing under a pressure of $20 \mathrm{MPa}$ for $5 \mathrm{sec}$, were measured with a CCD camera using SImage minisoftware. The contact angles were determined from the average of measurements at ten random points per sample (i.e., $n=10$ ). The crystalline phases of calcium carbonates were analyzed with an X-ray diffractometer (XRD) (PANalytical $\mathrm{X}^{\prime}$ pertMPD, Netherlands; $\mathrm{Cu} \mathrm{K} \alpha$ : $45 \mathrm{kV}, 40 \mathrm{~mA}$ ). 


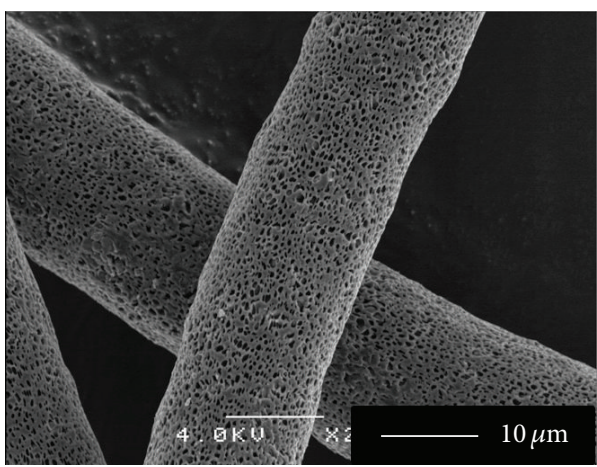

(a)

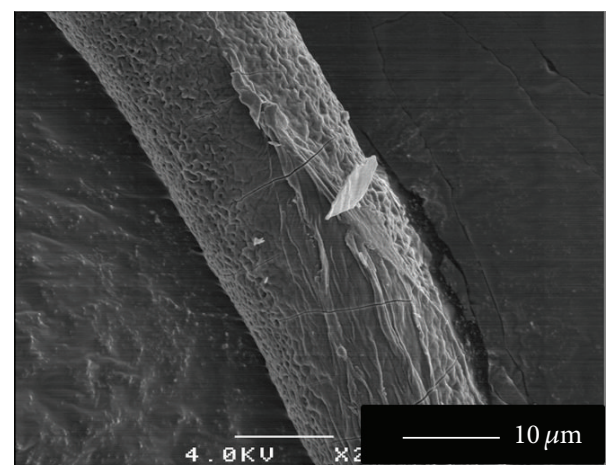

(b)

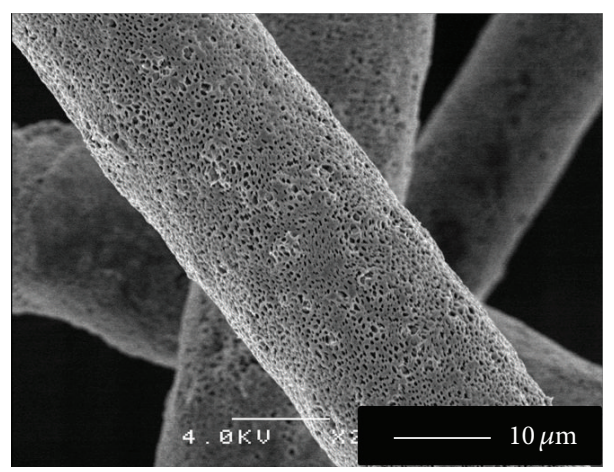

(c)

Figure 1: SEM images of (a) PLLA/SiV and ((b), (c)) INT-modified PLLA/SiV; (b) PLLA/SiV-INT(50-1) and (c) PLLA/SiV-INT(10-5).

Each sample of $10 \mathrm{mg}$ was soaked in $10 \mathrm{~mL}$ of Tris buffer solution ( $\mathrm{pH} \sim 7.4)$ at $37^{\circ} \mathrm{C}$ for $24 \mathrm{~h}$. The released calcium and silicate ion amounts from samples were measured by inductively coupled plasma-atomic emission spectroscopy (ICP-AES) (ICPS-7000, Shimadzu, Japan). Three specimens at each point were measured (i.e., $n=3$ ).

2.5. Cell Culture. Twenty milligrams of each sample was put in a well of 24-well plates and compressed with a silica glass tube of $15 \mathrm{~mm}$ outer diameter, $12 \mathrm{~mm}$ inner diameter, and $15 \mathrm{~mm}$ height. The resultant samples were sterilized using ethylene oxide gas. The samples were wetted by ethanol and then they were rinsed with a culture medium (alpha minimum essential medium, $\alpha$-MEM) containing $10 \%$ fetal bovine serum. Murine osteoblast-like (MC3T3-E1) cells were seeded onto the samples with a density of 200,000 cells per well and incubated $3 \mathrm{~h}$ at $37^{\circ} \mathrm{C}$ in $5 \% \mathrm{CO}_{2}$ for evaluation of their initial attachment. After the incubation, the samples were rinsed with $\alpha$-MEM and then incubated in the culture medium containing the reagent of the Cell Counting Kit8 (CCK-8; Dojindo, Japan) at $37^{\circ} \mathrm{C}$ for $2 \mathrm{~h}$. The attached cell number on samples was colorimetrically estimated by measuring the absorbance of the resulting medium at $450 \mathrm{~nm}$ $(n=3)$. Differences between the samples were determined by Student's $t$-test, with $P<0.05$, which is considered to be statistically significant. The samples after $3 \mathrm{~h}$ of the incubation were fixed with $2.5 \%$ glutaraldehyde for $40 \mathrm{~min}$ at $4^{\circ} \mathrm{C}$, dehydrated through a series of increasing concentrations of ethanol, and finally dried with hexamethyldisilazane. The morphology of attached cells on samples was observed by FESEM.

\section{Results and Discussion}

Figure 1 shows the fiber morphologies of cotton-like PLLA/ $\mathrm{SiV}$ before and after INT modification. The average and standard deviation of 100 parts of electrospun fiber diameters were 12 and $3 \mu \mathrm{m}$, respectively. Temperature and relative humidity are known as minor factors in the controlling of fiber diameter [25]. The temperature and relative humidity could affect the certain random fiber diameters of PLLA/SiV. As shown in Figure 1(a), the fibers have numerous pores of submicrometer diameter on their surfaces, due to the volatilization of the solvent during electrospinning. The fiber surface of PLLA/SiV-INT(50-1) was covered partially with the aggregated INTs, as shown in Figure 1(b). On the other hand, as shown in Figure 1(c), the numerous pores on the fiber surface of PLLA/SiV-INT(10-5) were observed; there was almost no change in the fiber morphology between the samples before and after 5 times of the dip-coating using highly diluted solution. INT-containing solution diluted with ethanol is considered to reduce the formation of INT aggregates. The aggregation rate of INTs maybe reduced in lower INTs concentration with an ethanol diluted solution. The EDS analysis (not shown here) exhibited the existence of aluminum, originated from INTs. The amount of detected 


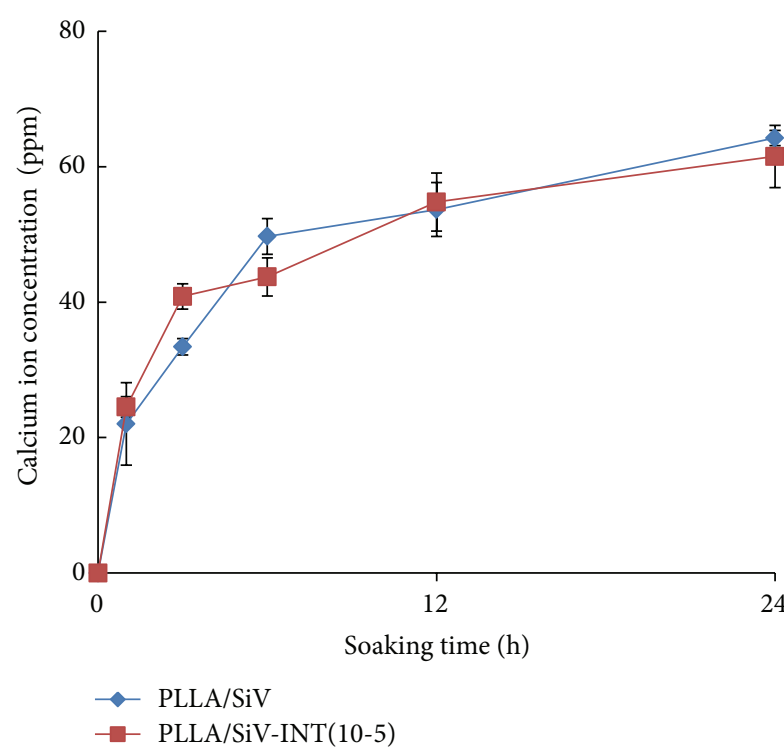

(a)

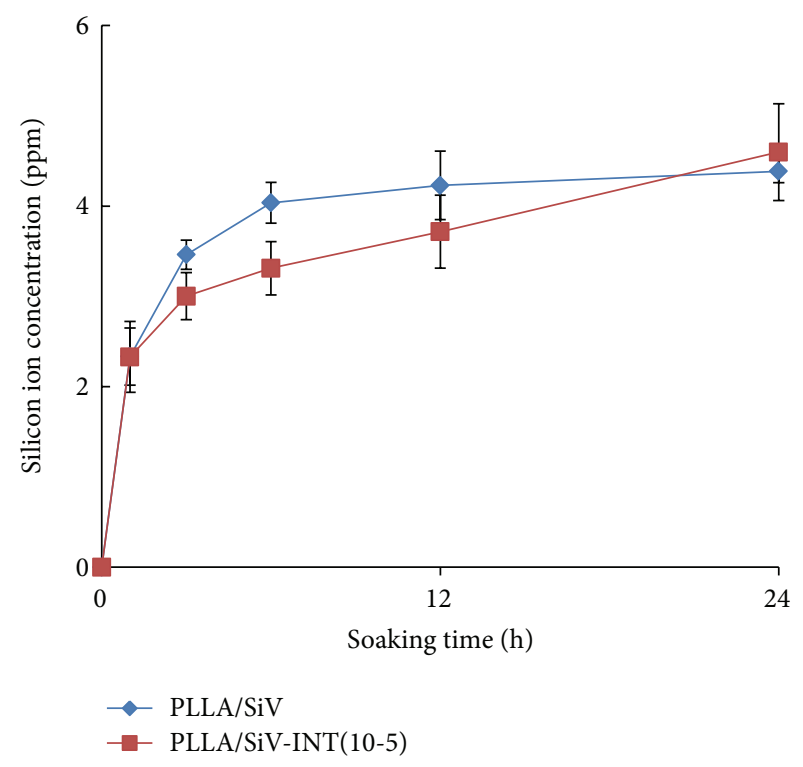

(b)

Figure 2: (a) Calcium and (b) silicon amounts released from PLLA/SiV and PLLA/SiV-INT(10-5) after soaking in Tris buffer solution. Circle: PLLA/SiV, diamond: PLLA/SiV-INT(10-5).

aluminum was $\sim 5.0 \pm 1.8$ at $\%$ in PLLA/SiV-INT(10-5). This result indicated that INTs were coated on PLLA/SiV fiber surfaces by dip-coating. The crystalline phase of calcium carbonate (vaterite) in cotton-like PLLA/SiV was identified from XRD analysis; the peak of calcite did not appear. Almost no differences in the XRD patterns of between samples before and after the modification were observed. No conversion of vaterite into calcite occurred during the repeated dip-coating process.

In EDS analysis of our previous report, almost no $\mathrm{Al}$ was detected on the fiber of fiber mats by dipping [20]. The hydrophobic surface of PLLA/SiV could be a cause of the difficulty to INT modifying because the INTs dispersed solution was based in water. The surface tension is a decisive factor to determine the wettability of a liquid on the solid surface [26]. Vázquez et al. reported that surface tension of the ethanol aqueous solution decreased with increasing the ratio of ethanol [27]. In case of ethanol diluting, the INTs aqueous solution could get high wettability with PLLA/SiV fibers surface. Thus, dip-coating with ethanol diluted INTs solution could be a useful method for INT modifying of the surface of cotton-like PLLA/SiV.

In contact angle measurement, the water drop penetrated into the INT-modified samples, immediately. In contrast, the contact angle of PLLA/SiV was $105^{\circ}$. INT modification was found to have an excellent effect on the improvement of the fibers in the cotton-like PLLA/SiV, owing to high water-absorption ability of the nanotube structure [28, 29]. As a result, the INT-modification makes it easier for the cell suspension to penetrate into the matrix. That is, we believe that there is no difference in the hydrophilicity between the INT-modified samples. PLLA/SiV-INT(10-5) was discussed to compare it with PLLA/SiV, hereafter.

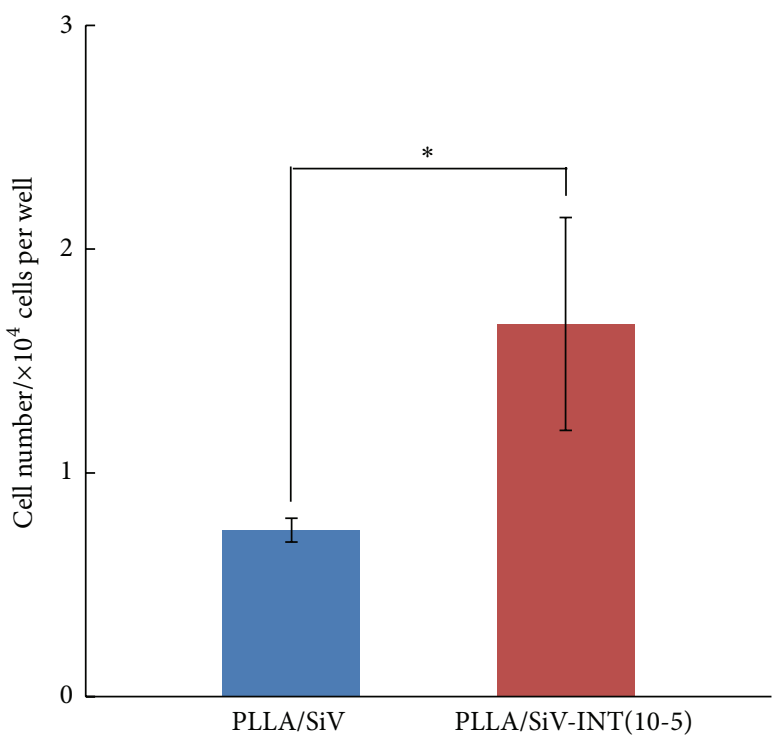

FIGURE 3: CCK-8 viability assay of MC3T3-E1 cells after $3 \mathrm{~h}$ of incubation on PLLA/SiV and PLLA/SiV-INT(10-5).

Figure 2 shows the released calcium and silicate ion amounts from samples. Both samples showed a similar trend on their releasing behaviors, independent of INT modification. It is supposed that the INT modification does not interrupt the calcium and silicate ions release from PLLA/SiV fibers.

Figure 3 shows attached cell numbers after $3 \mathrm{~h}$ of incubation on the samples. It is considerable that the great parts of seeded cells were passed through the cotton-like samples because the samples have very high porosity. The attached 


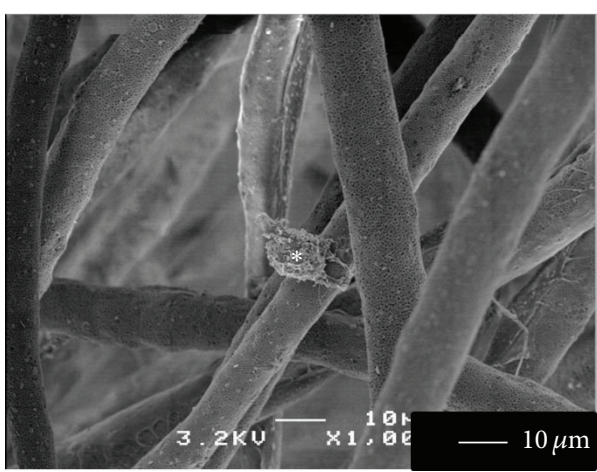

(a)

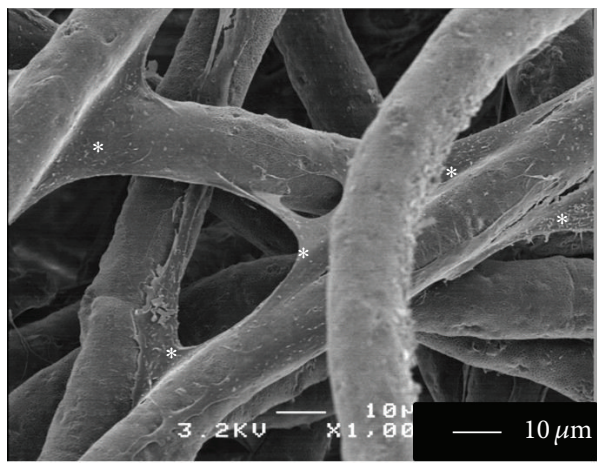

(c)

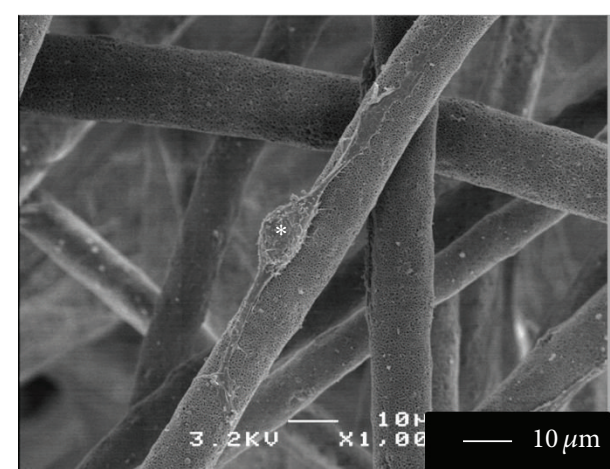

(b)

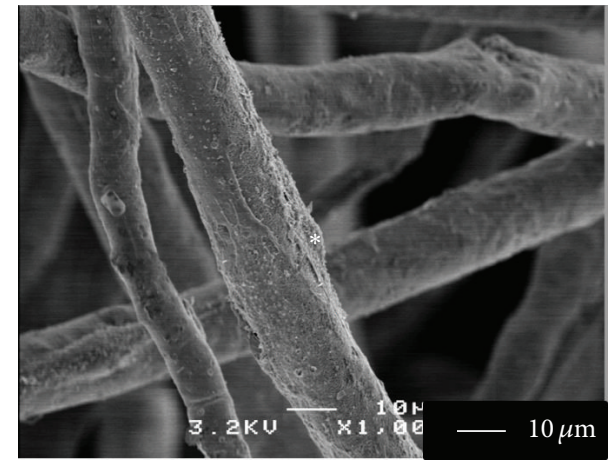

(d)

FIGURE 4: SEM images of MC3T3-E1 cells after $3 \mathrm{~h}$ of incubation on ((a), (b)) PLLA/SiV and ((c), (d)) PLLA/SiV-INT(10-5). Asterisk indicates the attached cells.

cell numbers on PLLA/SiV-INT(10-5) were almost twice of those on PLLA/SiV. Figure 4 exhibits the cell morphologies on the samples after $3 \mathrm{~h}$ of incubation. The cells on the PLLA/SiV were still spherical, as shown in Figures 4(a) and 4(b). In contrast, the cells on PLLA/SiV-INT(10-5) were more spread widely than those on PLLA/SiV. Some of them on PLLA/SiV-INT(10-5) were bridged between adjacent fibers. Aoki et al. reported that the well-spreaded cells adhered stronger and have more affinity to scaffold than round cells [30]. This suggests that the INT-modified cotton-like PLLA/SiV has more favorable surface for cell attachment than PLLA/SiV. Some reports suggest that the protein adsorption on the surface of materials contributes to cell adhesion $[31,32]$. In our preliminary experiment, imogolite showed high adsorption ability with cell-adhesive proteins such as fibronectin or vitronectin. The cell-adhesive proteins might be more easily adsorbed on the fiber surfaces of INT-modified cotton-like PLLA/SiV than those of the PLLA/SiV. Therefore, the evaluation on protein adsorption ability of INT-modified cotton-like PLLA/SiV is currently in progress.

\section{Conclusions}

The fiber surface of cotton-like PLLA/SiV was modified successfully with imogolite nanotubes by a dip-process using ethanol-diluted aqueous solution. There was almost no change in the fiber morphology even after the surface modification. The calcium and silicate ions releasing behavior of the modified sample was almost the same as that of PLLA/SiV. The cell adhesion on PLLA/SiV was enhanced by surface modification with imogolite. Thus, the surface modification may be useful for improving cellular compatibility of biodegradable polymer materials.

\section{Conflict of Interests}

The authors declare no competing financial interests.

\section{Acknowledgments}

The present work was supported in part by a Grant-in-Aid for Scientific Research from the Japan Society for the Promotion of Science (no. 25630282) and a Grant from the Institute of Ceramics Research and Education, NITech.

\section{References}

[1] J. C. Middleton and A. J. Tipton, "Synthetic biodegradable polymers as orthopedic devices," Biomaterials, vol. 21, no. 23, pp. 2335-2346, 2000.

[2] Y. Chen, S. Zhou, and Q. Li, "Microstructure design of biodegradable scaffold and its effect on tissue regeneration," Biomaterials, vol. 32, no. 22, pp. 5003-5014, 2011.

[3] P. Mainil-Varlet, B. Rahn, and S. Gogolewski, "Long-term in vivo degradation and bone reaction to various polylactides. 1 . One-year results," Biomaterials, vol. 18, no. 3, pp. 257-266, 1997. 
[4] A. Hoppe, N. S. Güldal, and A. R. Boccaccini, "A review of the biological response to ionic dissolution products from bioactive glasses and glass-ceramics," Biomaterials, vol. 32, no. 11, pp. 2757-2774, 2011.

[5] I. D. Xynos, A. J. Edgar, L. D. K. Buttery, L. L. Hench, and J. M. Polak, "Ionic products of bioactive glass dissolution increase proliferation of human osteoblasts and induce insulin-like growth factor II mRNA expression and protein synthesis," Biochemical and Biophysical Research Communications, vol. 276, no. 2, pp. 461-465, 2000.

[6] T. Wakita, J. Nakamura, Y. Ota, A. Obata, T. Kasuga, and S. Ban, "Effect of preparation route on the degradation behavior and ion releasability of siloxane-poly(lactic acid)-vaterite hybrid nonwoven fabrics for guided bone regeneration," Dental Materials Journal, vol. 30, no. 2, pp. 232-238, 2011.

[7] A. Obata, T. Hotta, T. Wakita, Y. Ota, and T. Kasuga, "Electrospun microfiber meshes of silicon-doped vaterite/poly(lactic acid) hybrid for guided bone regeneration," Acta Biomaterialia, vol. 6, no. 4, pp. 1248-1257, 2010.

[8] T. Wakita, A. Obata, G. Poologasundarampillai, J. R. Jones, and T. Kasuga, "Preparation of electrospun siloxane-poly(lactic acid)-vaterite hybrid fibrous membranes for guided bone regeneration," Composites Science and Technology, vol. 70, no. 13, pp. 1889-1893, 2010.

[9] T. Kasuga, A. Obata, H. Maeda, Y. Ota, X. Yao, and K. Oribe, "Siloxane-poly(lactic acid)-vaterite composites with 3D cottonlike structure," Journal of Materials Science, vol. 23, no. 10, pp. 2349-2357, 2012.

[10] A. Obata, H. Ozasa, T. Kasuga, and J. R. Jones, "Cotton wool-like poly (lactic acid)/vaterite composite scaffolds releasing soluble silica for bone tissue engineering," Journal of Materials Science, vol. 24, no. 7, pp. 1649-1658, 2013.

[11] P. B. Van Wachem, T. Beugeling, and J. Feijen, "Interaction of cultured human endothelial cells with polymeric surfaces of different wettabilities," Biomaterials, vol. 6, no. 6, pp. 403-408, 1985.

[12] Y. Tamada and Y. Ikada, "Cell adhesion to plasma-treated polymer surfaces," Polymer, vol. 34, no. 10, pp. 2208-2212, 1993.

[13] Y. Arima and H. Iwata, "Effect of wettability and surface functional groups on protein adsorption and cell adhesion using well-defined mixed self-assembled monolayers," Biomaterials, vol. 28, no. 20, pp. 3074-3082, 2007.

[14] P. Cradwick, V. Farmer, J. Russell, C. Masson, K. Wada, and N. Yoshinaga, "Imogolite, a hydrated aluminium silicate of tubular structure," Nature, vol. 240, no. 104, pp. 187-189, 1972.

[15] K. Ishikawa, S. Abe, Y. Yawaka, M. Suzuki, and F. Watari, “Osteoblastic cellular responses to aluminosilicate nanotubes, imogolite using Saos-2 and MC3T3-E1 cells," Journal of the Ceramic Society of Japan, vol. 118, no. 1378, pp. 516-520, 2010.

[16] K. Ishikawa, T. Akasaka, S. Abe, Y. Yawaka, M. Suzuki, and F. Watari, "Application of imogolite, almino-silicate nanotube, as scaffold for the mineralization of osteoblasts," Bioceramics Development and Applications, vol. 1, pp. 1-3, 2011.

[17] K. Ishikawa, T. Akasaka, Y. Nodasaka et al., "Physical properties of aluminosilicate nanotubes, imogolite, as scaffold and effect on osteoblastic mineralization," Nano Biomedicine, vol. 1, no. 2, pp. 109-120, 2009.

[18] A. Obata, S. Tokuda, and T. Kasuga, "Enhanced in vitro cell activity on silicon-doped vaterite/poly(lactic acid) composites," Acta Biomaterialia, vol. 5, no. 1, pp. 57-62, 2009.

[19] K. Fujikura, S. Lin, J. Nakamura, A. Obata, and T. Kasuga, "Preparation of eletrospun fiber mats using siloxane-containing vaterite and biodegradable polymer hybrids for bone regeneration," Journal of Biomedical Materials Research Part B, 2013.

[20] S. Yamazaki, H. Maeda, A. Obata, K. Inukai, K. Kato, and T. Kasuga, "Aluminum silicate nanotube coating of siloxane-poly (lactic acid)-vaterite composite fibermats for bone regeneration," Journal of Nanomaterials, vol. 2012, Article ID 463768, 7 pages, 2012.

[21] K. Fujikura, A. Obata, and T. Kasuga, "Cellular migration to electrospun poly (lactic acid) fibermats," Journal of Biomaterials Science, Polymer Edition, vol. 23, no. 15, pp. 1939-1950, 2012.

[22] M. S. Rao, "Kinetics and mechanism of the transformation of vaterite to calcite," Bulletin of the Chemical Society of Japan, vol. 46, pp. 1414-1417, 1973.

[23] N. Spanos and P. G. Koutsoukos, “The transformation of vaterite to calcite: effect of the conditions of the solutions in contact with the mineral phase," Journal of Crystal Growth, vol. 191, no. 4, pp. 783-790, 1998.

[24] S.-F. Chen, S.-H. Yu, J. Hang, F. Li, and Y. Liu, "Polymorph discrimination of $\mathrm{CaCO}_{3}$ mineral in an ethanol/water solution: formation of complex vaterite superstructures and aragonite rods," Chemistry of Materials, vol. 18, no. 1, pp. 115-122, 2006.

[25] S. De Vrieze, T. Van Camp, A. Nelvig, B. Hagström, P. Westbroek, and K. De Clerck, "The effect of temperature and humidity on electrospinning," Journal of Materials Science, vol. 44, no. 5, pp. 1357-1362, 2009.

[26] Y. Yuan and T. R. Lee, "Contact Angle and Wetting Properties," in Surface Science Techniques, G. Bracco and B. Holst, Eds., pp. 3-34, Springer, Berlin, Germany, 2013.

[27] G. Vázquez, E. Alvarez, and J. M. Navaza, "Surface tension of alcohol + water from 20 to $50^{\circ} \mathrm{C}$," Journal of Chemical and Engineering Data, vol. 40, no. 3, pp. 611-614, 1995.

[28] V. C. Farmer, M. J. Adams, A. R. Fraser, and F. Palmieri, "Synthetic imogolite: properties, synthesis, and possible applications," Clay Minerals, vol. 18, no. 4, pp. 459-472, 1983.

[29] M. Suzuki, "Absorption properties and application of allophane and imogolite," Journal of Clay Science Society of Japan, vol. 42, no. 3, pp. 144-147, 2003 (Japanese).

[30] N. Aoki, A. Yokoyama, Y. Nodasaka et al., "Strikingly extended morphology of cells grown on carbon nanotubes," Chemistry Letters, vol. 35, no. 5, pp. 508-509, 2006.

[31] T. A. Horbett and M. B. Schway, "Correlations between mouse 3T3 cell spreading and serum fibronectin adsorption on glass and hydroxyethylmethacrylate-ethylmethacrylate copolymers," Journal of Biomedical Materials Research, vol. 22, no. 9, pp. 763793, 1988.

[32] Y. Yang, R. Cavin, and J. L. Ong, "Protein adsorption on titanium surfaces and their effect on osteoblast attachment," Journal of Biomedical Materials Research A, vol. 67, no. 1, pp. 344349, 2003. 

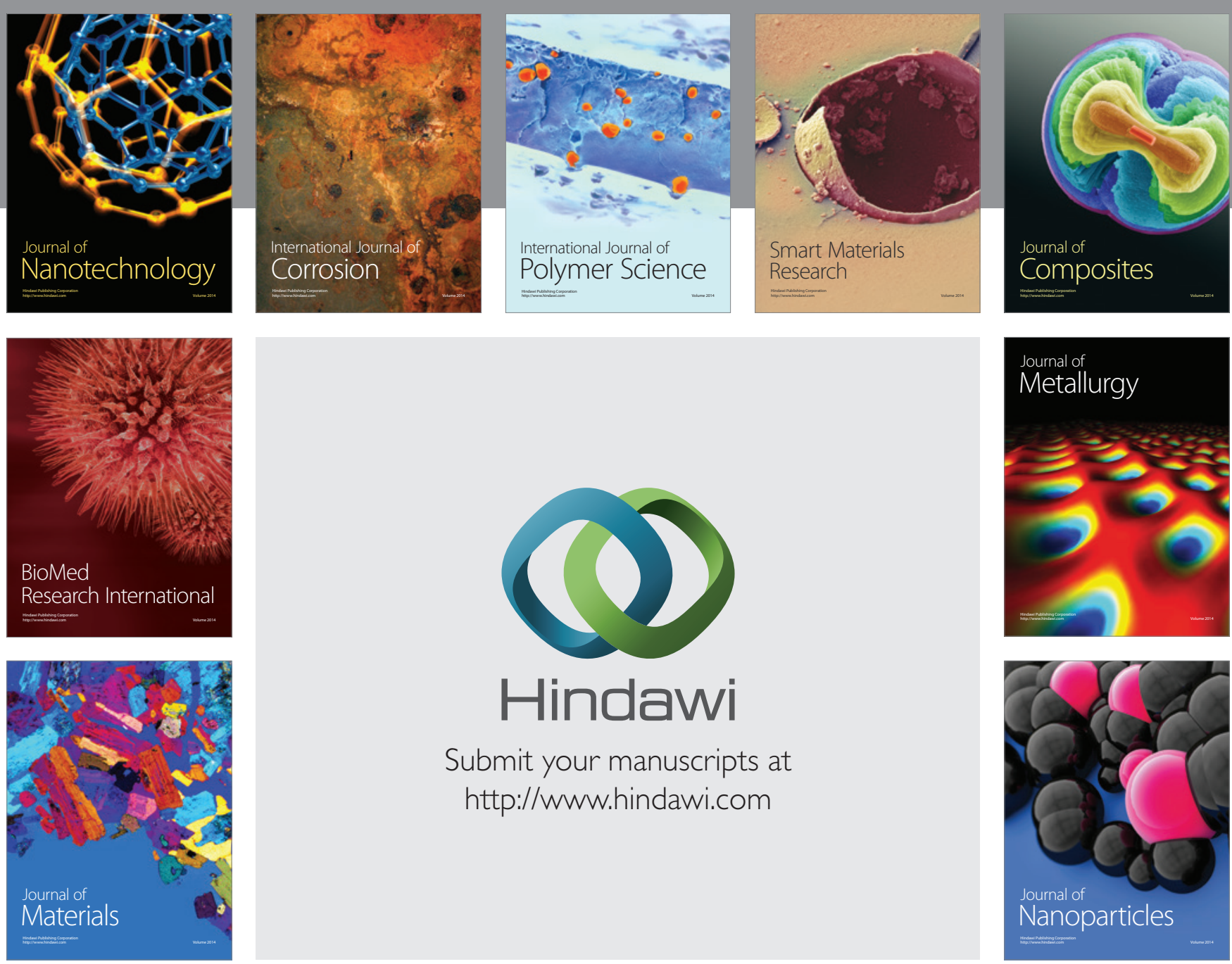

Submit your manuscripts at http://www.hindawi.com
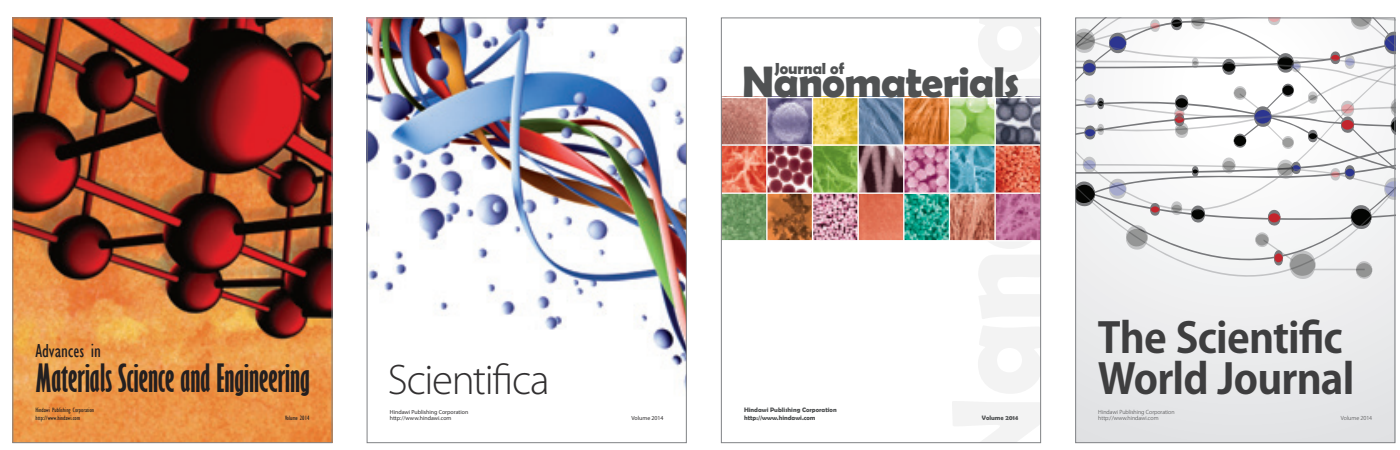

\section{The Scientific World Journal}
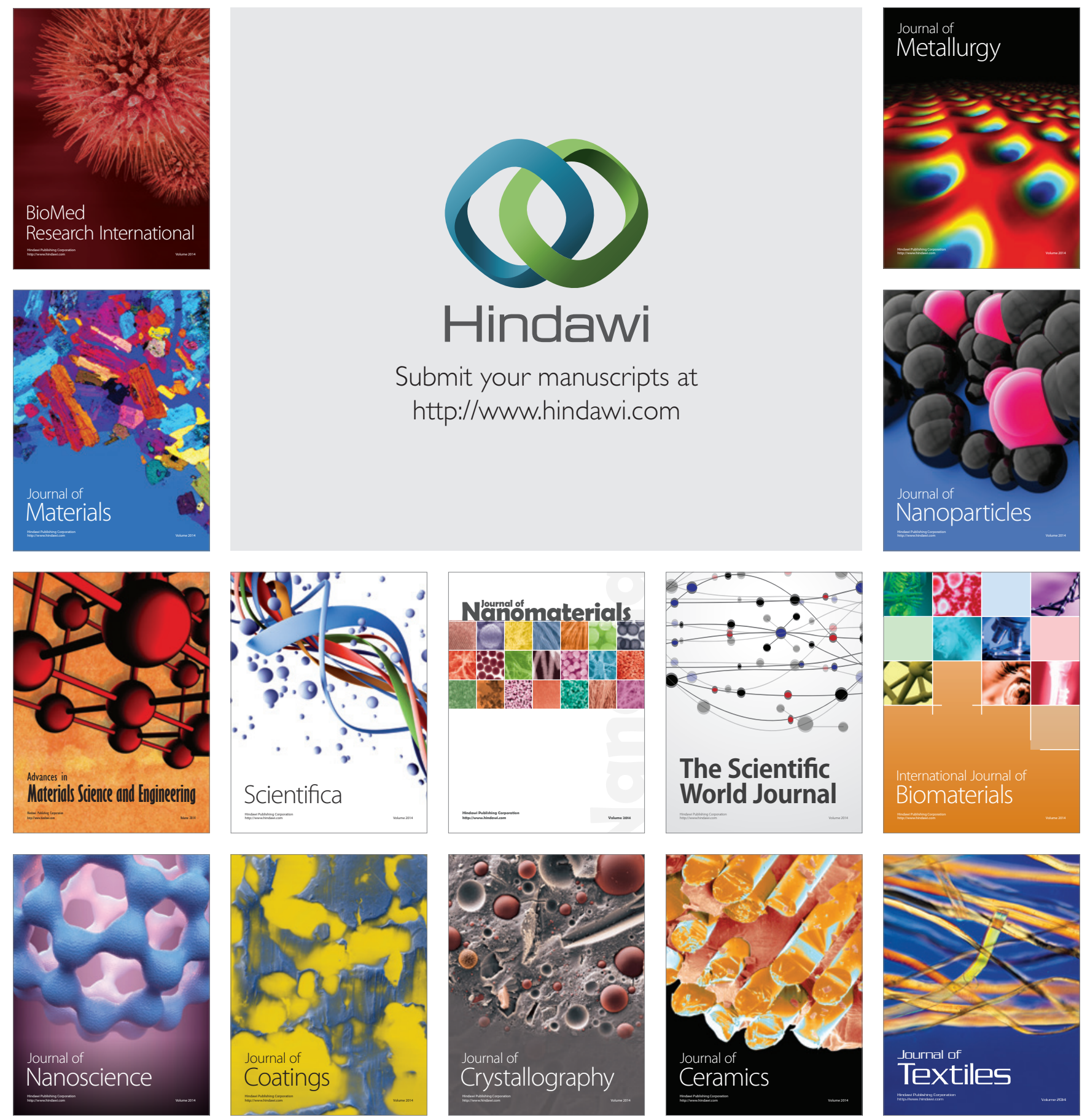